\title{
Automatic 3D segmentation of MRI data for detection of head and neck cancerous lymph nodes
}

\author{
Baixiang Zhao, John Soraghan, Gaetano \\ Di-caterina, and Lykourgos Petropoulakis \\ Centre for Signal and Image processing, \\ University of Strathclyde, \\ Glasgow,United Kingdom \\ baixiang.zhao@strath.ac.uk, \\ j.soraghan@strath.ac.uk, \\ gaetano.di-caterina@strath.ac.uk \\ 1.petropoulakis@strath.ac.uk
}

\author{
Derek Grose \\ Beatson West of Scotland Cancer Centre \\ Glasgow,United Kingdom \\ Derek.Grose@ggc.scot.nhs.uk
}

\author{
Trushali Doshi \\ Micrima Limited \\ Bristol, UK \\ trushalid@gmail.com
}

\begin{abstract}
A novel algorithm for automatic 3D segmentation of magnetic resonance imaging (MRI) data for detection of head and neck cancerous lymph nodes $(\mathrm{LN}))$ is presented in this paper. The proposed algorithm pre-processes the MRI data slices to enhance quality and reduce artefacts. A modified Fuzzy c-mean process is performed through all slices, followed by a probability map which refines the clustering results, to detect the approximate position of cancerous lymph nodes. Fourier interpolation is applied to create an isotropic 3D MRI volume. A new 3D level set method segments the tumour from the interpolated MRI volume. The proposed algorithm is tested on synthetic and real MRI data. The results show that the novel cancerous lymph nodes 3D volume extraction algorithm has over 0.9 Dice similarity score on synthetic data and 0.7 on real MRI data. The F-measure is 0.92 on synthetic data and 0.75 on real data.
\end{abstract}

Keywords-MRI data, Head and neck cancer, Modified fuzzy cmean, probability map, 3D level set method (LSM)

\section{INTRODUCTION}

Radiotherapy, along with surgery, provides the main option for curative cancer treatment. The delineation of cancerous lymph nodes can help determine the clinical target volume (CTV) [1]. CTV is the exact tumour volume with margin for subclinical microscopic spread and affected lymph node. Definition of this region is fundamental for accurate and effective radiation treatment planning. Development of automated delineation methods can help reduce inter and intra variabilities of manual tumour delineation, providing objective and reliable assistance to clinical oncologists to reduce work load and improve radiation treatment [2].

Fig. 1(a) shows a T1 weighted gadolinium-enhanced head and neck MR image with cancerous lymph nodes. It is known that cancerous lymph nodes have fuzzy boundaries and they are not significantly distinct from neighbour tissues. Furthermore as seen in Fig. 1(b) artefacts of MRI data, such as uneven illumination, are present. All these make automatic segmentation of cancerous areas a very challenging task.

A variety of algorithms have been proposed for cancerous lymph nodes segmentation, such as training based approaches
[3], graph cut techniques [4], and deformable models [5, 6]. These previous works mostly segment lymph nodes from CT images in 2D, and some approaches rely on manual segmentation. On the contrary, the novel work in this paper addresses the automatic delineation of cancerous lymph nodes from a series of MRI slices, using a 3D level set method (LSM).

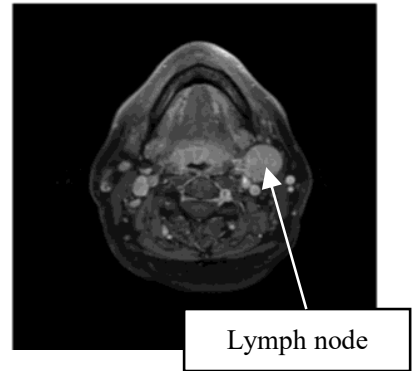

(a)

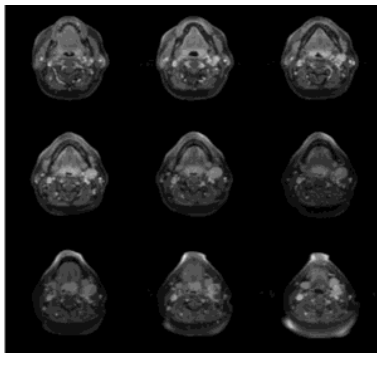

(b)
Fig. 1. (a) A T1-weighted Gadolinium-enhanced head and neck MR image example with cancerous lymph nodes; (b) A image serires from one MRI dataset.

This paper presents a new fully automatic algorithm for 3D segmentation of cancerous lymph nodes volume from T1weighted Gadolinium-enhanced MRI data. The challenges of this work include segmenting tumour regions with fuzzy boundaries, non-uniform intensities, and avoiding adjacent anatomical structures. It is essential to determine the position of lymph nodes, and the intensity range of the cancerous lymph node area is very important. This novel algorithm is validated on both synthetic and real MRI data from the Beatson West of Scotland Cancer Centre, in Glasgow.

The remainder of the paper is organised as follows. Section 2 describes the new automatic cancerous lymph nodes 3D segmentation algorithm. Section 3 demonstrates the experimental results on both synthetic and read MRI datasets. The last section summaries the paper.

\footnotetext{
* Research supported by Beatson Cancer Charity.
} 


\section{AUTOMATIC HEAD AND NECK ABNORMAL LYMPH NODES 3D SEGMENTATION}

The proposed head and neck cancerous lymph nodes segmentation is shown in Fig. 2. The algorithm contains two main parts: a) Image pre-processing, b) Cancerous lymph nodes $3 \mathrm{D}$ segmentation.

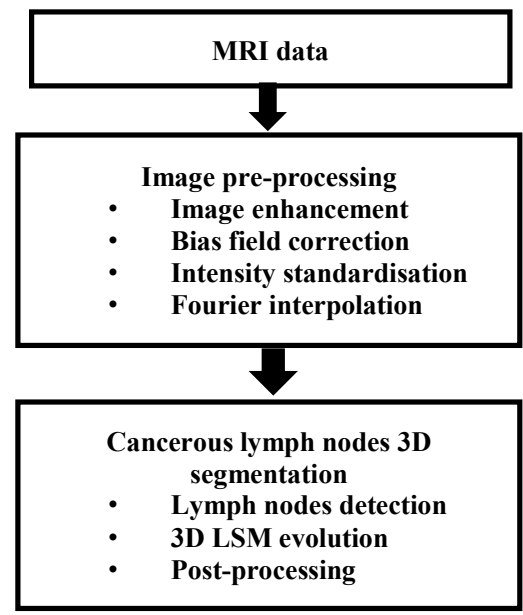

Fig. 2. Flowchart of automatic cancerous lymph nodes segmentation.

\section{A. Image pre-processing}

In this work, multiple pre-processing techniques are applied to the MRI data for artefacts removing and image enhancing. Background noise is minimised using morphological opening and a majority operation [7], which can remove small noisy regions while preserving edges in the image. The images are enhanced using a background brightness preserving contrast enhancement technique [8]. There are two types of intensity variations in MRI data. The first type is intensity inhomogeneity (IIH) (also named as Bias field) [9] on a single slice. In this paper, IIH is estimated and corrected based on techniques described in [10]. The second type of intensity variation is between slices. This occurs when a certain measured intensity cannot be associated with a specific tissue class [9] on all slices. In this work the effect of intensity variation is reduced using an intensity standardisation between slices[7]. The MRI data used in this work has anisotropic voxels, while 3D LSM only works well on isotropic voxels. Original voxels are converted to isotopic voxels through Fourier interpolation, which was introduced in [11]. The volume for LSM segmentation is reconstructed in 3D using both real and interpolated slices.

\section{B. Cancerous lymph nodes detection}

Results of the level set evolution rely on the initialisation and target intensity range setting. The initialisation provides a start point (initial seed) for the level set method, and sets the size of the initial seed. The target intensity range is a rough estimate of the range of pixel intensities of the cancerous lymph node region. The detection of lymph nodes helps determine start point of level set evolution, and the intensity range of cancerous lymph nodes area. The steps for lymph nodes detection are shown in Fig. 3.

Throat detection $\Rightarrow$ Modified FCM $\Rightarrow$ Probability map

Fig. 3. Workflow for lymph nodes detection.

Cancerous lymph nodes detection is performed slice by slice. For each MRI slice, the throat is detected by two fuzzy rules [2]. Then a modified fuzzy c-mean (MFCM) algorithm [12] utilises intensity and spatial information of pixels to organise them into five clusters. Clustering into five categories is based on the assumption that a pre-processed head and neck MRI slice consists of four main tissue types (fatty tissue, cancer tissue, normal tissue, and normal muscle tissues) and background. Based on prior biomedical knowledge from clinicians, head and neck cancerous regions (tumour and cancerous LN) are normally located around the throat region, and they usually have first or second brightest intensity among all tissues.

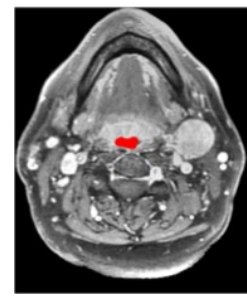

(a)
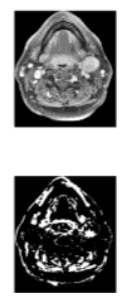
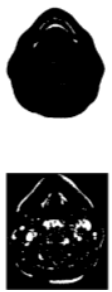

(b)
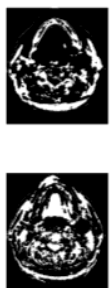

Fig. 4. (a) A pre-processed MRI slice with detected throat region (red is); (b) Modified fuzzy c-mean, left top is original image, other five are five clusters, from left to right first row, then left to right second row, the intensity of each cluster is brighter.

Fig. 4(a) illustrates the typical throat detection result. Based on the throat location and intensities of pixels, the MFCM groups pixels into five clusters. The cancerous lymph nodes are in first or second brightest clusters (bottom right and bottom middle shown in Fig. 4(b)). Regions of interest are taken from these clusters and combined with edge information of the original image to separate large regions. Morphological opening is used to further separate regions, and small regions are removed.

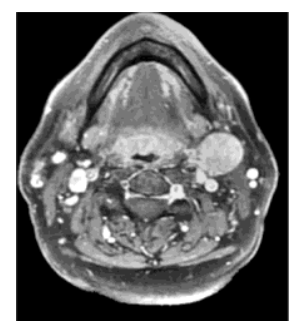

(a)

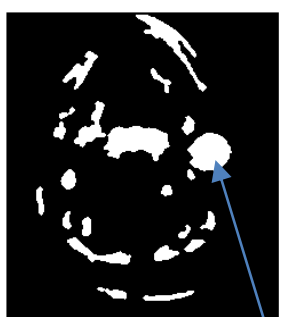

(b) Lymph node
Fig. 5. (a) A pre-processed MRI slice;(b) Clustering result after removing small noisy regions.

The resulting regions are shown in Fig. 5(b), where the lymph node is identifiable.

To roughly detect the position of the cancerous lymph node, a probability map, $W$, of lymph nodes location is proposed to 
identify a region from Fig. 5(b). This region has the highest probability of overlapping with lymph node.

$$
W=\alpha W_{\text {location }}+\beta W_{\text {size }}+\theta W_{\text {eccentricity }}
$$

Eq. 1 shows the components of the probability map $W$. There are three parts on the right hand side of (1). The location weight, $W_{\text {location }}$, is the weight factor derived from the location of regions compared to throat. The second component is $W_{\text {size }}$, i.e. the 'size weight'. This factor is set for de-weighting large regions. If a region is not in an expected lymph node position but has a large area, this region is likely to be a false positive in the lymph node detection scheme. Thus, $W_{\text {size }}$ is used, based on the idea that the location of region centre should be more emphasised during the detection. This means that the location of a region's centre is far more important than other points inside this region. The last component of the probability map is $W_{\text {eccentricity. }}$ This is derived from the region's eccentricity, according to the prior knowledge that cancerous lymph nodes which are mostly circular shapes. In (1), $\alpha, \beta$ and $\theta$ are parameters controlling the contribution of the three parts, and are so that $\alpha+\beta+\theta=1$.

The construction of $W_{\text {location }}$ may be written as:

$$
\begin{gathered}
W_{\text {location }}=w_{c} \cap \\
w_{c}=\left\{\begin{array}{cc}
0 & \left|C_{n}-y\right|<a \\
\frac{\left|C_{n}-y-a\right|}{|a-b|} & a<\left|C_{n}-y\right|<b \\
1-\frac{\left|C_{n}-y-b\right|}{|c-b|} & b<\left|C_{n}-y\right|<c \\
0 & \text { else }
\end{array}\right. \\
w_{r}=\left\{\begin{array}{cc}
1-m \frac{R_{n}-x}{g} & \left|R_{n}-x\right|<g \\
(1-m)-\frac{\left|R_{n}-x-g\right|}{|h-g|} & g<\left|R_{n}-x\right|<h \\
0 & \text { else }
\end{array}\right.
\end{gathered}
$$

where $R_{n}$ and $C_{n}$ are row and column coordinates,.. $w_{c}$ is the weight derived from column position, and $w_{r}$ is derived from row position. Parameters $x$ and $y$ are the centroid coordinates of the throat region. Parameters $a-c, g$ and $h$ are adaptive thresholds which are determined based on whether a pixel is in a close-to-throat region or away-from-throat region as illustrated in Fig. 6(a) and (b). The parameter $m$ is a control coefficient which ranges from 0 to 1 . It is adaptively set based on the ratio between throat width and image width.

The intersection symbol at the top of (2) denotes the fuzzy AND operator. The values of $a-h$ are adaptive to the throat region's size, and they are automatically set based on throat region's width and height. For example, if on an MRI slice the throat height is less than 0.1 of image height, $a$ will be set as 0.8 of throat height. Fig. 6 shows probability maps of two MRI slices. It also displays positions of $a$ - $h$ in these two maps. In (2), $a-c$ are the horizontal coordinates of red lines $a-c$ in Fig. $6 ; g-h$ in (2) are the vertical coordinates of the blue lines $g-h$ in Fig. 6. Pixels located between line $a$ and $b$ or line $b$ and $c$ will have different location weights.

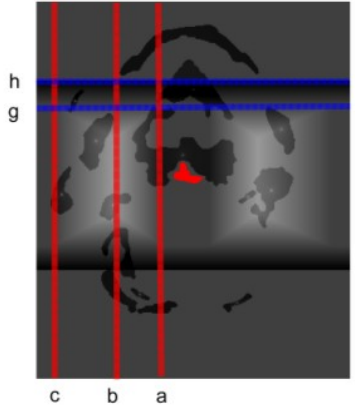

(a)

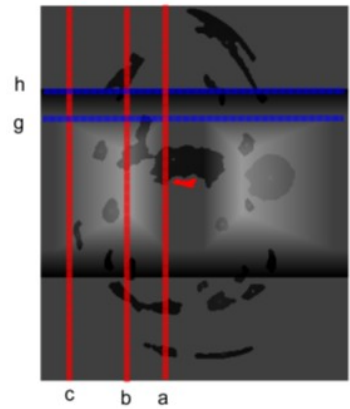

(b)
Fig. 6. Probability map and demonstration of thresholds. Red lines with texts show position of a-c, and blue lines with texts show position of g-h. Red regions in (a) and (b) show positions of throat region. (a)(b) only display one side of a-h on single slice, a-h are symmetry on the other side of same slice.

The value of $W_{\text {location }}$ is set according to the prior knowledge that the head and neck lymph nodes are located at two sides of the throat region but not closely adjacent to the throat.

Assuming that a region has $n$ points then the size weight of each region may be calculated as follows:

$$
W_{\text {size }}=\sum_{i=1}^{n} \frac{1}{\sqrt{\left(x_{i}-x_{c}\right)+\left(y_{i}-y_{c}\right)}+e p s}
$$

where $x_{i}$ and $y_{i}$ are the coordinates of $i_{t h}$ point; $x_{c}$ and $y_{c}$ are the coordinates of the region centre; eps is a small positive number (e.g. machine epsilon) to ensure non division by zero. In this case, if a region has a large size, but its centre is actually located at an undesired area, this region will not be picked by the algorithm. Thus false positives will be less likely generated in the detection section.

Eccentricity is calculated as follows:

$$
W_{\text {eccentricity }}=\frac{C}{A}
$$

where $\mathrm{C}$ is the distance from the region centre to the focus along the major axis of ellipse, and $\mathrm{A}$ is the length of major axis. When eccentricity is close to 0 , the region is likely to be a circle; when it is close to 1 , the region is likely to be elongated.

From the probability map $W$ for the processed MRI slice Fig. 5(a) shown in Fig. 7(a), it can be seen that two sides of throat (but not adjacent to throat) have the highest probability. After overlapping the probability map with regions in Fig. 5(b), the region which has the highest probability to be lymph node is determined. This is displayed in Fig. 7(b) with a red mark.

In each slice, one region is detected to be most likely overlapped with a lymph node area. Fig. 9(a) shows the detection results of all slice in a 3D view. If a slice has no cancerous lymph nodes, a false positive region may be detected, such as the left bottom blob shown in Fig. 9(a). Thus, a further processing step (Fig. 8) is added to remove false positives, and also determine $3 \mathrm{D}$ location of cancerous lymph nodes. 


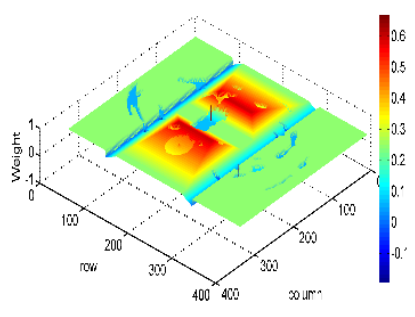

(a)

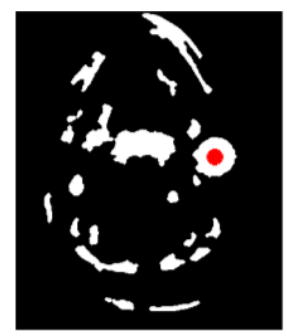

(b)
Fig. 7. (a) Probability map of Fig. 5(a); (b) Regions and detected rough lymph nodes position marked with red point.

Fig. 8 shows the section of the proposed algorithm which removes the false positives from the lymph node rough detection process, and thus determines the 3D position of lymph nodes. Firstly, the detected regions are sorted based on their radii. Second, the region with largest radius $R$ and centre $(X, Y)$ is taken; other regions whose centres is inside $(X+R \cos \theta$, $Y+R \sin \theta)$ are assigned to the same group. Then the process is repeated on those regions which have not been grouped, until all regions are grouped. The search process is performed slice by slice. Then after this process, all regions are clustered into groups. The group which contains the majority of regions is kept, and regions in other groups are discarded; thus regions overlapped with cancerous lymph node are all detected. The lymph node centre can be calculated by taking the average of the regions' coordinates.

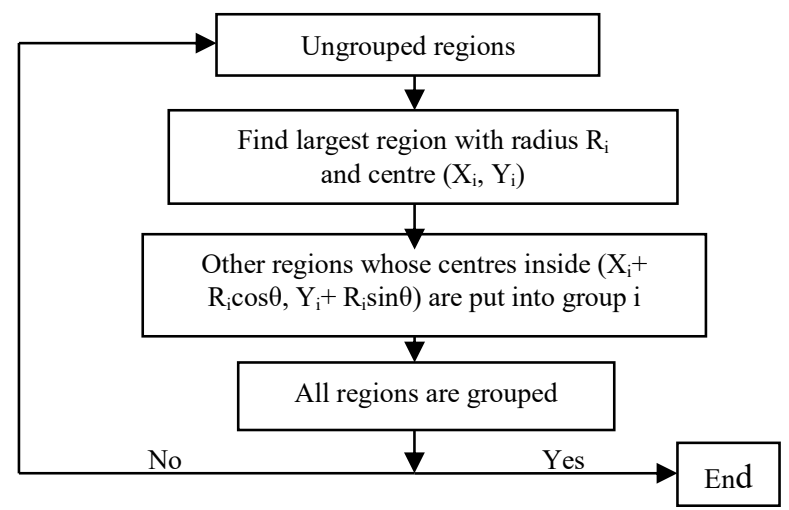

Fig. 8. Work flow for grouping detected regions based on their horizontal location.

\section{Lymph nodes segmentaion with 3D LSM}

The initial seed for the $3 \mathrm{D}$ level set function is set at the centre of the detected lymph node. The horizontal size of the initial seed is 0.05 of the interpolated image stack's horizontal size. The height of seed is one third of rough lymph node height. An example of initial seed is shown in Fig. 9(b).

In this work, the speed function $\mathrm{F}$ used for $3 \mathrm{D}$ level set evolution is [13]:

$$
\begin{gathered}
F=\lambda(\varepsilon-|I(x, y, z)-T|)+(1-\lambda) \nabla \cdot \frac{\nabla \varphi}{|\nabla \varphi|} \\
=F_{e x t}+F_{i n t}
\end{gathered}
$$

where $\nabla \frac{\nabla \phi}{|\nabla \phi|}$ is the average curvature of evolving curve; $T$ is mean intensity of detected lymph nodes region; $\varepsilon$ is standard deviation of all pixels inside the detected lymph nodes region, and $\alpha$ is a weighting factor $0<\lambda<1$. The right hand side of (5) comprises an external force $F_{\text {ext }}$, which drives the curve to the boundary and an internal force $F_{\text {int }}$, that keeps segmentation result smooth. This level set function is based on intensities of pixels, and curvature of evolving curve.

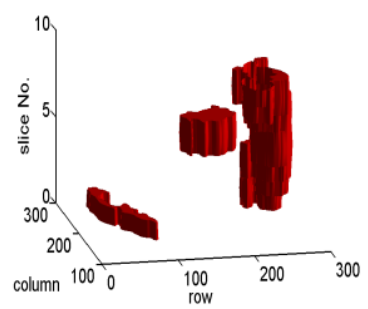

(a)

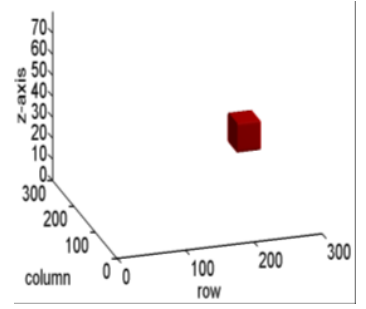

(b)
Fig. 9. (a) Regions detected by probability map; (b) Initial seed for 3D LSM.

Some visual results from the 3D LSM segmentation are shown in Fig. 10. Fig. 10(a-c) shows the evolution process and (d) is the 3D LSM result. It can be seen from Fig. 10(c) that there are unsmooth surfaces. These are due to weak boundaries of lymph nodes region, and some adjacent tissues that have similar intensities. Thus, post-processing is applied. The postprocessing uses 3D morphological operations to remove the unsmooth parts. The 3D morphological operation applied comprises $3 \mathrm{D}$ erosion first to remove noise and separate $3 \mathrm{D}$ objects; then the largest connected component is picked among $3 \mathrm{D}$ objects; finally $3 \mathrm{D}$ dilation is applied to compensate the volume loss in erosion. The final segmentation result is shown in Fig. 10(d).

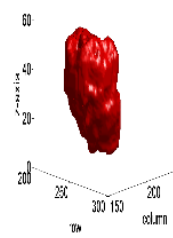

(a)

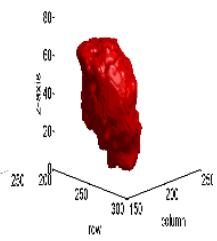

(b)

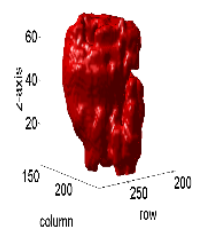

(c)

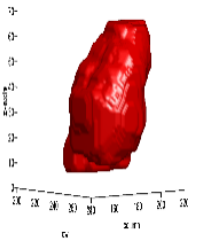

(d)
Fig. 10. (a) shows the LSM evolution result after 200 iterations, (b) shows the result after 300 iterations, and (c) is the final LSM result (after 500 iterations). (d) shows 3D segmentaion after post-processing.

\section{EXPERIMENTAL RESULTS}

The new algorithm was implemented in Matlab, running on a PC with 16G RAM, 3.2GHz Intel(R) Core(TM) i7-8700 CPU. Experiments were conducted on both synthetic and real MRI datasets.

\section{A. Experiments on synthetic data}

A synthetic MRI dataset was generated using a modified Shepp and Logan head phantom function from [14]. In this work, the generated synthetic data has 8 slices, and three configurable parameters to add artefacts, including contrast 
reduction, bias field, and noise. In our experiment, the added artefacts include Rician noise [14] with standard deviation of 10 , bias field (IIH) generated by cos function [14], and decreased LN-to-background contrast with ratio at 0.3 .

Results on synthetic dataset are measured by Dice similarity coefficient (DSC). The DSC is to measure similarity between

$$
\operatorname{DSC}(A, B)=\frac{2 \times(A \cap B)}{A+B}
$$

two samples A and B; it can be calculated as given [15].

From Fig. 11, experiments using synthetic data shows that the proposed algorithm has tolerance to artefacts, and the automatic segmentation is highly overlapped with the ground truth.

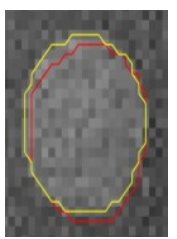

(a)

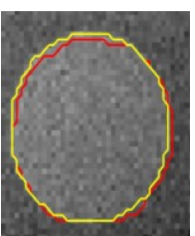

(b)

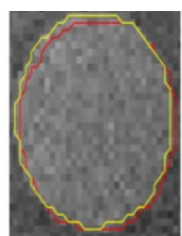

(c)

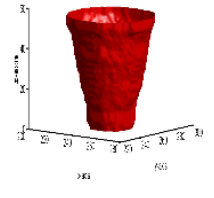

(d)
Fig. 11. Simulation on sythetic datasets with Red contours automatic segmentation, and Yellow contours ground truth (a) result from bottom slice (b) result from middle slice (c) result fromtop slice. (d) automatic extracted 3D LN.

Fig. 12 shows that the DSCs between automatic segmentation $(A$ in (6)) and ground truth $(B$ in (6)) are around $90 \%$, and the mean DSC is $91 \%$. The F-measure of automatic segmentation result is 0.92 . This synthetic dataset has 8 slices, and the overall processing time was 152 seconds.

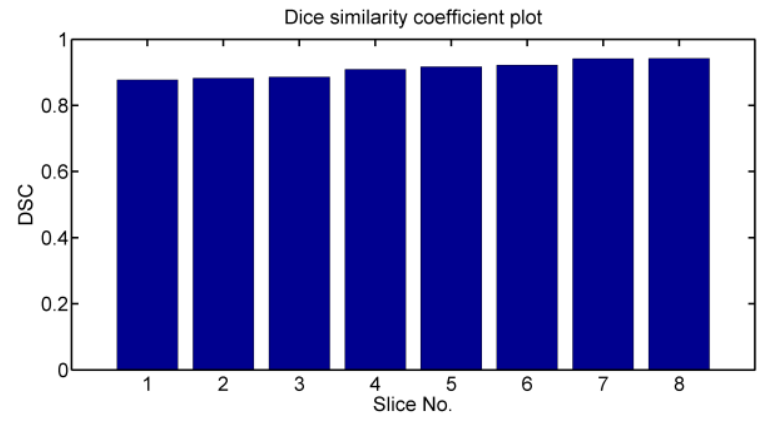

Fig. 12. Dice similarity score for each slice of a sythetic MRI dataset. The xaxis shows slice number, and y-axis shows DSC score.

\section{B. Experiments on real data}

The proposed algrotihms was tested on 5 real datasets (each one has on average 10 slices) from Beatson West of Scotland Cancer Centre, in Glasgow. Results in this section demonstrate some extracted 3D cancerous lymph nodes, and their contours on 2D slices. Also, the automatic segmentations are compared with a consensus tumour outline. The consensus tumour outlines on 2D axial slices were formed by clinicians from Beatson West of Scotland Cancer Centre.
Fig. 13 to Fig. 15 shows examples of extracted 3D cancerous lymph nodes, and also extracted 2D contours with annotated consensus outline. It can be seen in Fig. 13-15 that the cancerous lymph nodes in Dataset1-3 are well extracted. Fig. 13(a)(b)(c) show that even when lymph node boundaries are very similar to adjacent tissues, the algorithm can still track the lymph node and segment the cancerous region. Furthermore, Fig. 13-15 shows that the proposed algorithm provides similar segmentation results as compared to the consensus outline.

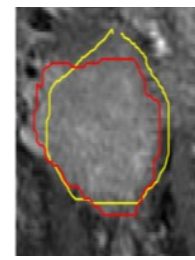

(a)

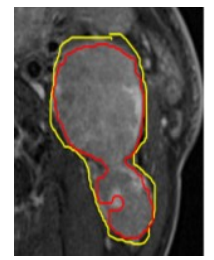

(b)

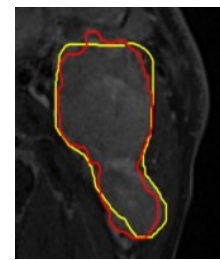

(c)

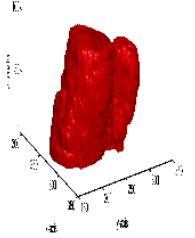

(d)
Fig. 13. Segmented cancerous lymph nodes from Dataset1. Red contours are automatic segmenation, and yellow contours are gold standards (consensu manual outline) (a) 2D contours of top slice (b) 2D contours of the middle slice, (c) 2D contours of the bottom slice (d) 3D visualisation of lymph node.

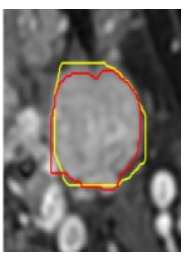

(a)

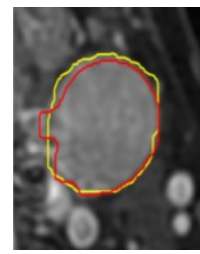

(b)

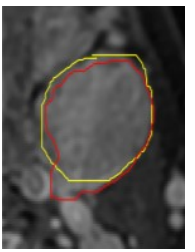

(c)

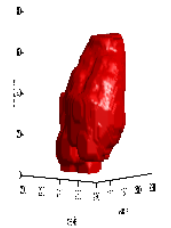

(d)
Fig. 14. Segmented cancerous lymph nodes from Dataset 2. Red contours are automatic segmenation, and yellow contours are gold standards (consensu manual outline) (a) 2D contours of top slice (b) 2D contours of the middle slice (c) 2D contours of the bottom slice (d) 3D visualisation of lymph node.

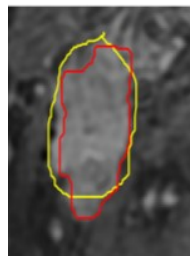

(a)

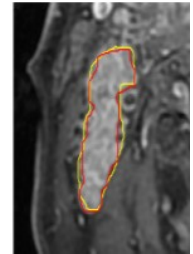

(b)

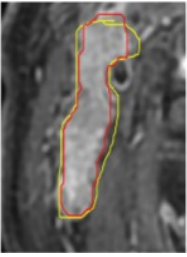

(c)

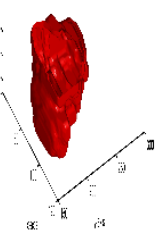

(d)
Fig. 15. Segmented cancerous lymph nodes from Dataset 3. Red contours are automatic segmenation, and yellow contours are gold standards (consensu manual outline) (a) 2D contours of top slice (b) 2D contours of the middle slice, (c) 2D contours of the bottom slice. (d) 3D visualisation of lymph node.

The proposed algorithm |was quantitatively assessed using the Dice similarity coefficient (DSC) and F-measure. DSC measures the overlapping rate between two areas. Fig. 16 shows the DSC of between automatic segmentation and consensus outlines..

As the boxplot in Fig. 16 illustrates, the medians of DSCs on 5 datasets are all above $60 \%$. From left to right in Fig. 16, the first dataset has highest DSC 90\%, dataset 3 and 5 have DSCs around $80 \%$, dataset 2 has DSC around $70 \%$, and dataset 5 has lowest DSC $60 \%$. The mean DSC through five datasets is 
$70 \%$. The average false negative rate is 0.0025 , and the average false positive rate is 0.2023 .

As bar graph shown in Fig. 17 illustrates, three datasets have F-measure score around 0.8 and higher, dataset 2 has Fmeasure score about 0.7 , and dataset 5 has lowest F-measure score of 0.54 . The mean F-measure score through 5 datasets is 0.75 . Each dataset has 8-10 MRI slices; the average processing time of proposed algorithm on each dataset is 250 seconds, and the processing time on each slice is 30 seconds.

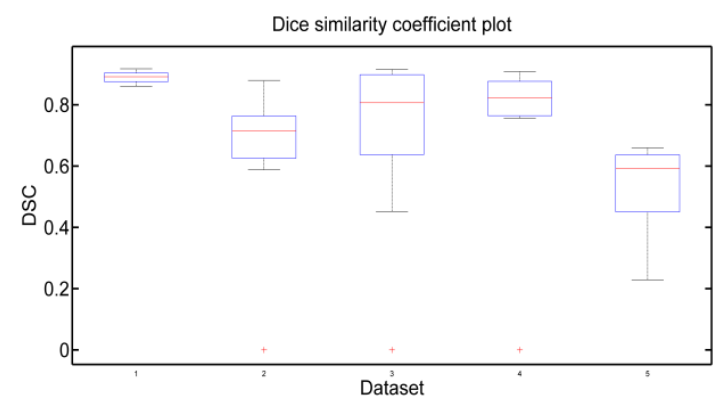

Fig. 16. Dice similarity coefficient on 5 head and neck MRI datasets.

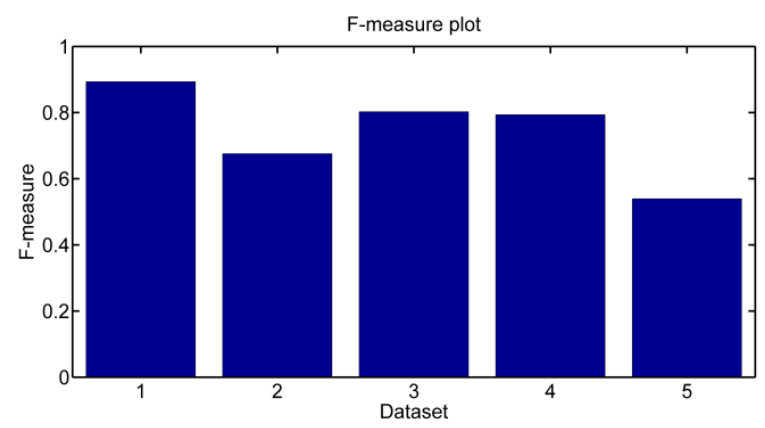

Fig. 17. F-mearsure on 5 head and neck MRI datasets.

\section{CONCLUSION}

This paper presented a new algorithm for automatic detection, 3D segmentation, and visualisation of cancerous lymph nodes from T1-weighted Gadolinium-enhanced head and neck MR images. The proposed method was demonstrated to work well on both synthetic and real MRI datasets. The results on synthetic data show that this method is tolerant to artefacts. The results on real data show that this algorithm can segment most tumour regions and can produce smooth surfaces.

In the future, this method will be tested on more MRI datasets. Also a quantitative study will be taken on more annotated data. The modification on de-noising methods, and the involvement of shape constrained 3D level set function are also objectives for future study.

\section{ACKNOWLEDGMENT}

The authors would like to acknowledge grant from Beatson Cancer Charity for their financial support with this study.

\section{REFERENCES}

[1] N. G. Burnet, S. J. Thomas, K. E. Burton, and S. J. Jefferies, "Defining the tumour and target volumes for radiotherapy," Cancer Imaging, vol. 4, pp. 153-161, 2004.

[2] T. Doshi, J. Soraghan, L. Petropoulakis, G. Di Caterina, D. Grose, K. MacKenzie, and C. Wilson, "Automatic pharynx and larynx cancer segmentation framework (PLCSF) on contrast enhanced MR images," Biomedical Signal Processing and Control, vol. 33, pp. 178-188, 2017/03/01/2017.

[3] J. Feulner, S. K. Zhou, M. Huber, J. Hornegger, D. Comaniciu, and A. Cavallaro, "Lymph node detection in 3-D chest CT using a spatial prior probability," in 2010 IEEE Computer Society Conference on Computer Vision and Pattern Recognition, 2010, pp. 2926-2932.

[4] Y. Wang and R. Beichel, "Graph-Based Segmentation of Lymph Nodes in CT Data," in Advances in Visual Computing, Berlin, Heidelberg, 2010, pp. 312-321.

[5] J. Yan, T.-g. Zhuang, B. Zhao, and L. H. Schwartz, "Lymph node segmentation from CT images using fast marching method," Computerized Medical Imaging and Graphics, vol. 28, pp. 33-38, 2004/01/01/ 2004.

[6] J.-Y. Zhou, W. Fang, K.-L. Chan, V. F. H. Chong, and J. B. K. Khoo, "Extraction of Metastatic Lymph Nodes from MR Images Using Two Deformable Model-based Approaches," Journal of Digital Imaging, vol. 20, pp. 336-346, 2007.

[7] R. C. Gonzalez, "Digital image processing," ed: Prentice hall, 2016.

[8] T. Tan, K. Sim, and C. P. Tso, "Image enhancement using background brightness preserving histogram equalisation," Electronics letters, vol. 48 , pp. $155-157,2012$.

[9] F. Jager and J. Hornegger, "Nonrigid Registration of Joint Histograms for Intensity Standardization in Magnetic Resonance Imaging," IEEE Transactions on Medical Imaging, vol. 28, pp. 137-150, 2009.

[10] O. Salvado, C. Hillenbrand, Z. Shaoxiang, and D. L. Wilson, "Method to correct intensity inhomogeneity in MR images for atherosclerosis characterization," IEEE Transactions on Medical Imaging, vol. 25, pp. 539-552, 2006.

[11] S. Campbell, T. Doshi, J. Soraghan, L. Petropoulakis, G. D. Caterina, D. Grose, and K. MacKenzie, "3-dimensional throat region segmentation from MRI data based on fourier interpolation and 3dimensional level set methods," in 2015 37th Annual International Conference of the IEEE Engineering in Medicine and Biology Society (EMBC), 2015, pp. 2419-2422.

[12]T. Doshi, J. Soraghan, D. Grose, K. MacKenzie, and L. Petropoulakis, "Modified fuzzy c-means clustering for automatic tongue base tumour extraction from MRI data," in 2014 22nd European Signal Processing Conference (EUSIPCO), 2014, pp. 2460-2464.

[13] A. E. Lefohn, J. M. Kniss, C. D. Hansen, and R. T. Whitaker, "A streaming narrow-band algorithm: interactive computation and visualization of level sets," IEEE Transactions on Visualization and Computer Graphics, vol. 10, pp. 422-433, 2004.

[14] R. V. D. Walle, H. H. Barrett, K. J. Myers, M. I. Aitbach, B. Desplanques, A. F. Gmitro, J. Cornelis, and I. Lemahieu, "Reconstruction of MR images from data acquired on a general nonregular grid by pseudoinverse calculation," IEEE Transactions on Medical Imaging, vol. 19, pp. 1160-1167, 2000.

[15] T. Sørensen, " \{A method of establishing groups of equal amplitude in plant sociology based on similarity of species and its application to analyses of the vegetation on Danish commons\}," Biol. Skr., vol. 5, pp. 1-34, 1948 . 